Generalized displacement correlation method for estimating stress intensity factors

P. Fu, S. M. Johnson, R. R. Settgast, C. R. Carrigan

September 29, 2011

Engineering Fracture Mechanics 
This document was prepared as an account of work sponsored by an agency of the United States government. Neither the United States government nor Lawrence Livermore National Security, LLC, nor any of their employees makes any warranty, expressed or implied, or assumes any legal liability or responsibility for the accuracy, completeness, or usefulness of any information, apparatus, product, or process disclosed, or represents that its use would not infringe privately owned rights. Reference herein to any specific commercial product, process, or service by trade name, trademark, manufacturer, or otherwise does not necessarily constitute or imply its endorsement, recommendation, or favoring by the United States government or Lawrence Livermore National Security, LLC. The views and opinions of authors expressed herein do not necessarily state or reflect those of the United States government or Lawrence Livermore National Security, LLC, and shall not be used for advertising or product endorsement purposes. 


\section{Generalized displacement correlation method for estimating stress intensity factors}

Pengcheng Fu*, Scott M. Johnson, Randolph R. Settgast, and Charles R. Carrigan

Atmospheric, Earth, and Energy Division, Lawrence Livermore National Laboratory, 7000 East Avenue, L-286, Livermore, CA 94550

Summary:

Conventional displacement-based methods for estimating stress intensity factors require special quarter-point finite elements in the first layer of elements around the fracture tip and substantial near-tip region mesh refinement. This paper presents a generalized form of the displacement correlation method (the GDC method), which can use any linear or quadratic finite element type with homogeneous meshing without local refinement. These two features are critical for modeling dynamic fracture propagation problems where locations of fractures are not known $a$ priori. Because regular finite elements' shape functions do not include the square-root terms, which are required for accurately representing the near-tip displacement field, the GDC method is enriched via a correction multiplier term. This paper develops the formulation of the GDC method and includes a number of numerical examples, especially those consisting of multiple interacting fractures. We find that the proposed method using quadratic elements is accurate for mode-I and mode-II fracturing, including for very coarse meshes. An alternative formulation using linear elements is also demonstrated to be accurate for mode-I fracturing, and acceptable mode-II results for most engineering applications can be obtained with appropriate mesh refinement, which remains considerably less than that required by most other methods for estimating stress intensities.

Keywords: fracture mechanics, stress intensity factor, displacement correlation method, quarterpoint element, fracture propagation, fracture interaction

\section{Introduction}

The stress intensity factor (SIF) is an important concept in fracture mechanics for relating stress and energy release rate at the fracture tip to loading and crack geometry. Although closed-form analytical solutions are available for a number of special fracture-load configurations (many of which have been compiled in [1]), SIF's are often calculated in the context of numerical methods, especially the finite element method (FEM) for arbitrary fracture-load configurations. 
Several methods are available for calculating or estimating SIF's with the FEM, such as the Jintegral [2] and its variants, the stiffness derivative technique [3], and a suite of methods based on the interpretation of near-tip nodal displacements. In the last category, there are at least three variants, including the displacement extrapolation method [4-7], the quarter-point displacement method [8], and the displacement correlation method [9,10]. These methods and others have been compared in a number of studies [e.g. 5,11-14]. One of the most significant advantages of the displacement-based methods is the simple formulation. Although the displacement-based methods were often found to be less accurate than the J-integral or the stiffness derivative method under certain conditions, the accuracy remains acceptable for most engineering applications [e.g. 5,14]. Many of the displacement-based methods were developed in the 1970's and 1980's in tandem with various special "quarter-point" finite element types [15-19] used in these methods. Though few new developments have been made for the displacement-based methods in the intervening decades [20], they continue to be widely used.

Displacement-based SIF calculation methods usually requires the following two conditions: 1) Quarter-point elements must be used in the first layer of elements around the crack tip; and 2) the mesh in the near-tip region has to be substantially refined for reasonable accuracy. This study seeks to relax these constraints of the displacement-based methods to accommodate analysis of a wider range of engineering systems, including hydraulic fracturing with explicitly coupled geomechanics-discrete fracture flow modeling [21]. The simulation of hydraulic fracturing serves as the motivating example for this paper. A hydraulic fracturing process usually involves modeling multiple cracks propagating along arbitrary paths, so the locations of the crack tips are not specified a priori. . If special quarter-point elements and refined mesh were required for each tip, the cost of remeshing would quickly overwhelm available computational resources.

Moreover, the implementation of the J-integral or the stiffness derivative method in such an explicitly coupled Lagrangian hydromechanical simulator is impractical for similar reasons. The generalized displacement correlation (GDC) method proposed here enables practical simulation of the hydraulic fracturing process by relaxing the mesh and element restriction of the conventional displacement-base methods yet inheriting the simplicity and computational efficiency.

We first review the mechanical and mathematical principles behind the original displacementbased methods in a generalized context in section 2. Compared with the original derivation of these methods, the loading condition is generalized by including crack surface traction and the meshing scheme is generalized by circumventing the dependency on the specific shape functions of quarter-point elements. This new GDC formulation encompasses the original formulation based on quarter-point elements as a special case. Subsequently, we develop the new 
generalized formulation in section 3 and further enhance its accuracy in section 4 by introducing an empirical correction multiplier term. In section 5, we test the new method against a number of fracture-load configurations with an emphasis on cases with inter-crack interactions, a situation critical to our hydraulic fracturing simulator development effort. The numerical examples in sections 4 and 5 use the same Poisson's ratio and near-tip mesh configuration. The sensitivity of the Poisson's ratio and near-tip mesh configurations are evaluated in section 6 .

\section{Review of displacement-based methods in a generalized framework}

Consider the two-dimensional (2D) continuum (linearly elastic and isotropic) around a crack tip as shown in Figure 1, with far-field normal $\left(\sigma_{f}\right)$ and shear $\left(\tau_{f}\right)$ stress existing along with crack surface traction $\left(\sigma_{c}\right.$ and $\left.\tau_{c}\right)$. Note that "traction" in this paper refers to stress distributed along fracture surface while the same term is often used in cohesive zone models for a different meaning. Stresses $\sigma_{f}, \tau_{f}, \sigma_{c}$, and $\tau_{c}$ are independent of each other, but the spatial variation of each of them is not considered. Their values can be either positive or negative, with the arrows in Figure 1 indicating the positive stress directions. According to the superposition principle, the mechanical response of this system is the sum of the responses of the three cases [(a) to (c)] to the right of the equal sign in the figure. Case (a) and case (b) respectively correspond to the classical boundary/loading conditions for mode-I and mode-II fracturing, whereas in case (c) the crack surface traction balances the far-field stress. Only the stress conditions in the two former cases [(a) and (b)] induce stress/strain singularities in the near-tip region, while the latter case (c) generates homogeneous stress and displacement fields which contribute to the overall mechanical response but not the near-tip stress sigularity. The loading conditions in case (a) and case (b) are the symmetric and skew-symmetric (antisymmetric) parts of the load that induce a near-tip stress singularity, respectively. Much of the development of fracture mechanics disregards the tractions along the crack surface, so case (a) and case (b) have been the focus of previous studies. In certain applications such as hydraulic fracturing, the pressure inside the fractures is the main mechanism for driving fracture extension with $\sigma_{c}<\sigma_{f}<0$. Under such conditions, the stress condition in case (c) significantly contributes to the mechanical responses of the system and cannot be overlooked. 


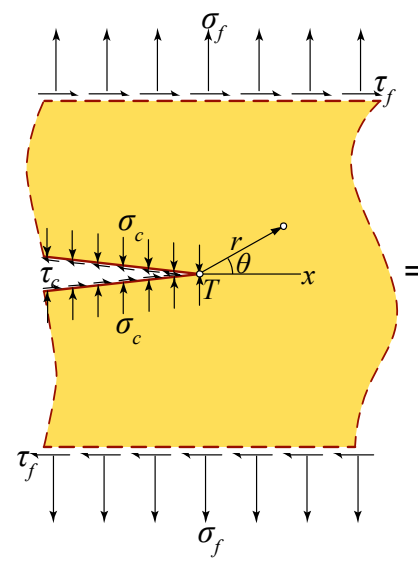

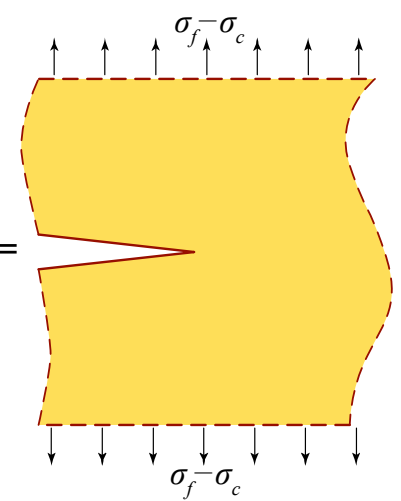

(a) Mode-I

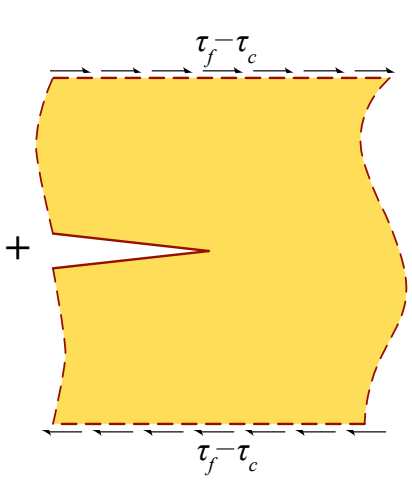

(b) Mode-II

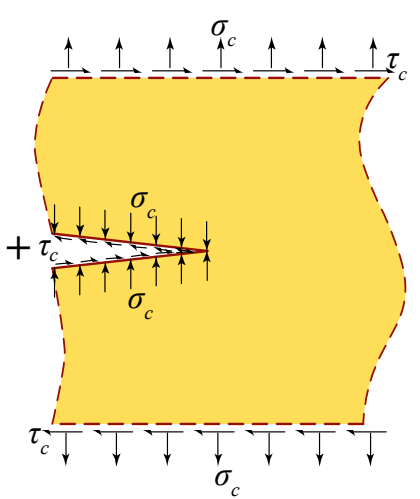

(c) Non-singular stress field

Figure 1 The near-tip region of a 2D medium and the separation of fracture modes according to the superposition principle. The polar coordinate system used in this study is denoted in the figure. Fracture openings in this and other examples are exaggerated for illustration purposes.

With higher-order terms omitted, the displacement field (relative to the crack tip displacement) induced by loading case (a) is

$$
\left\{\begin{array}{c}
u_{r}^{a} \\
u_{\theta}^{a}
\end{array}\right\}=\frac{K_{I}}{G} \sqrt{\frac{r}{2 \pi}}\left\{\begin{array}{c}
\cos \frac{\theta}{2} \\
-\sin \frac{\theta}{2}
\end{array}\right\}\left(\beta-\cos ^{2} \frac{\theta}{2}\right)
$$

where $K_{I}$ is the mode-I stress intensity factor; $G$ is the shear modulus of the medium; $\beta$ is a constant depending on whether this is a plane strain $(\beta=2[1-v]$ with $v$ being the Poisson's ratio) or a plane stress $(\beta=2 /[1+v])$ problem. It we assume that the elasticity parameters $(G$ and $\beta)$ are constants for a given problem, the equation can be simplified as

$$
\left\{\begin{array}{l}
u_{r}^{a} \\
u_{\theta}^{a}
\end{array}\right\}=K_{I} \sqrt{r}\left\{\begin{array}{l}
f_{r}^{a}(\theta) \\
f_{\theta}^{a}(\theta)
\end{array}\right\}
$$

where $f_{r}^{a}(\theta)$ and $f_{\theta}^{a}(\theta)$ are functions of the angular coordinate $(\theta)$ of the point where the displacement is measured. The effects of the elasticity parameters are incorporated into these two functions and they are considered constants for the purpose of this section. We can also write the corresponding equations for case (b), namely mode-II fracturing as

$$
\left\{\begin{array}{c}
u_{r}^{b} \\
u_{\theta}^{b}
\end{array}\right\}=\frac{K_{I I}}{G} \sqrt{\frac{r}{2 \pi}}\left\{\begin{array}{c}
-\sin \frac{\theta}{2}\left(\beta-3 \cos ^{2} \frac{\theta}{2}\right) \\
-\cos \frac{\theta}{2}\left(\beta+2-3 \cos ^{2} \frac{\theta}{2}\right)
\end{array}\right\}=K_{I I} \sqrt{r}\left\{\begin{array}{l}
f_{r}^{b}(\theta) \\
f_{\theta}^{b}(\theta)
\end{array}\right\}
$$


Loading in Figure 1(c) induces a homogeneous stress field quantified by $\sigma_{c}, \sigma_{x}$, and $\tau_{c} . \sigma_{x}$ is the normal stress component (not denoted in Figure 1) in the direction along the fracture tip, and is typically not concerned in fracture mechanics. The displacement induced by this homogeneous stress field is

$$
\begin{aligned}
& \left\{\begin{array}{l}
u_{r}^{c} \\
u_{\theta}^{c}
\end{array}\right\}=r\left\{\begin{array}{l}
f_{r}^{c}\left(\theta, \sigma_{c}, \sigma_{x}, \tau_{c}\right) \\
f_{\theta}^{c}\left(\theta, \sigma_{c}, \sigma_{x}, \tau_{c}\right)
\end{array}\right\} \\
& \text { or }\left\{\begin{array}{l}
u_{r}^{c} \\
u_{\theta}^{c}
\end{array}\right\}=r\left\{\begin{array}{l}
f_{r}^{c}(\theta) \\
f_{\theta}^{c}(\theta)
\end{array}\right\}
\end{aligned}
$$

for any known stress state $\left(\sigma_{c}, \sigma_{x}, \tau_{c}\right)$. The explicit expression of functions $f_{r}^{c}$ and $f_{\theta}^{c}$ can be derived based on Hooke's law, but it requires knowledge of the stress state and is not pursued here. Note that the $f^{c}$ terms also encompass the effects of small rigid-body rotation of the system, but this is not explicitly discussed in the following development. The most important implication of equation (5) for the scope of this paper is that along any "ray" direction originating from the fracture tip, the displacement of any point relative to that of the tip is linearly proportional to its distance to the crack tip under the homogeneous stress condition. Combining equations (2), (3), and (5), we can write the overall displacement field for the arbitrary loading condition in Figure 1 as

$$
\left\{\begin{array}{l}
u_{r} \\
u_{\theta}
\end{array}\right\}=\sqrt{r}\left\{\begin{array}{l}
f_{r}^{a} K_{I}+f_{r}^{b} K_{I I} \\
f_{\theta}^{a} K_{I}+f_{\theta}^{b} K_{I I}
\end{array}\right\}+r\left\{\begin{array}{l}
f_{r}^{c} \\
f_{\theta}^{c}
\end{array}\right\}
$$

with $K_{I}$ and $K_{I I}$ being the unknowns while $u_{r}$ and $u_{\theta}$ can be obtained from FEM solutions. In order to apply any displacement-based stress intensity calculation method, the medium containing the fracture needs to be modeled using a finite element mesh. Quarter-point elements, with the inverse square root singularity embedded by shifting the mid-edge nodes on the ray edges to the quarter-points, are usually employed as the first layer of elements around the tip as shown in Figure 2. Displacements along the crack face $(\theta=\pi)$ at nodes $A$ and $B$ are obtained by solving the finite element model. Noticing that $f_{r}^{a}(\pi)=0$ and $f_{\theta}^{b}(\pi)=0$, we have

$$
\begin{aligned}
u_{r}^{A} & =\frac{1}{2} \sqrt{l_{E}} f_{r}^{b}(\pi) K_{I I}+\frac{1}{4} l_{E} f_{r}^{c}(\pi) \\
u_{r}^{B} & =\sqrt{l_{E}} f_{r}^{b}(\pi) K_{I I}+l_{E} f_{r}^{c}(\pi) \\
u_{\theta}^{A} & =\frac{1}{2} \sqrt{l_{E}} f_{\theta}^{a}(\pi) K_{I}+\frac{1}{4} l_{E} f_{\theta}^{c}(\pi) \\
u_{\theta}^{B} & =\sqrt{l_{E}} f_{\theta}^{a}(\pi) K_{I}+l_{E} f_{\theta}^{c}(\pi)
\end{aligned}
$$


where $l_{E}$ is the length of the element edge ( $l_{E}=|T B|=4|T A|$ in this particular case). By applying basic linear equation manipulation/solving techniques, we can eliminate the terms involving $f_{r}^{c}$ or $f_{\theta}^{c}$ and obtain

$$
K_{I}=\frac{4 u_{\theta}^{A}-u_{\theta}^{B}}{\sqrt{l_{E}} f_{\theta}^{a}(\pi)} \text { and } K_{I I}=\frac{4 u_{r}^{A}-u_{r}^{B}}{\sqrt{l_{E}} f_{r}^{b}(\pi)}
$$

which is the core formulation for the displacement correlation method. The symmetry of the system can be exploited to improve the accuracy of the results with

$$
K_{I}=\frac{4\left(u_{\theta}^{A}-u_{\theta}^{A^{\prime}}\right)-\left(u_{\theta}^{B}-u_{\theta}^{B^{\prime}}\right)}{2 \sqrt{l_{E}} f_{\theta}^{a}(\pi)} \text { and } K_{I I}=\frac{4\left(u_{r}^{A}-u_{r}^{A^{\prime}}\right)-\left(u_{r}^{B}-u_{r}^{B^{\prime}}\right)}{2 \sqrt{l_{E}} f_{r}^{b}(\pi)}
$$

The formulation for the so-called quarter-point displacement method

$$
K_{I}=\frac{u_{\theta}^{A}-u_{\theta}^{A^{\prime}}}{\sqrt{l_{E}} f_{\theta}^{a}(\pi)} \text { and } K_{I I}=\frac{u_{r}^{A}-u_{r}^{A^{\prime}}}{\sqrt{l_{E}} f_{r}^{b}(\pi)}
$$

is valid only if the terms involving $f_{r}^{c}$ and $f_{\theta}^{c}$ in equation (6) vanish, implying the loading of the system is the sum of case (a) and case (b) excluding case (c) in Figure 1, i.e. there is no traction along the crack faces. This limitation of the quarter-point method was described by Tracey [10] but has largely been neglected, as it does not apply to the typical loading conditions in mechanical engineering, where crack surface tractions are absent. Although this limitation of the quarter-point displacement method does not lead to inaccuracies in many studies comparing these two methods in the context of mechanical engineering [12,13,19,22], it is highly deleterious if the method is to be used for hydraulic fracturing modeling or similar problems. The displacement extrapolation method suffers similarly since the loading scenario shown in case (c) of Figure 1 is not supported in the assumptions underlying that method. Based on this, we select the displacement correlation method as the basis for further development.

The original development of the displacement correlation method and the quarter-point displacement method derive the same equations as equations (11) and (12), respectively, through a different procedure. The purpose of the above development is to provide the necessary basis for the development of the new generalized method in the next section. 


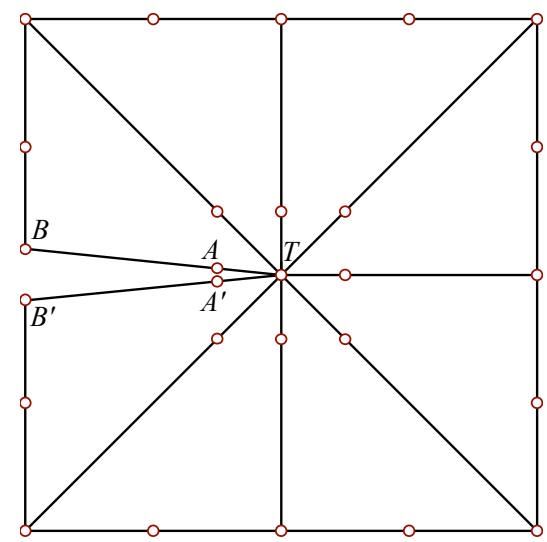

Figure 2 Quarter-point element configurations near a crack tip.

3. Formulation of the generalized method

From the procedure in section 2, we see that the core of the displacement correlation method is to solve equations of the following form

$u_{i}=f_{i}^{a} \sqrt{r_{i}} K_{I}+f_{i}^{b} \sqrt{r_{i}} K_{I I}+r_{i} f_{i}^{c}$

where $u_{i}, f_{i}^{a}$, and $f_{i}^{b}$ are known from FEM solutions of the specific fracture-load configuration and near-tip region closed-form solutions; $K_{I}$ and $K_{I I}$ are the two unknowns to solve; $f_{i}^{c}$ can be removed by the following procedure. Because $f_{i}^{c}$ is a function of the angular coordinate $\theta$ but not the radial coordinate $r$, we can use known displacements (either $u_{r}$ or $u_{\theta}$ ) and other information $\left(r_{i}, f_{i}^{a}\right.$, and $\left.f_{i}^{b}\right)$ at two points with the same angular coordinate $\theta$ to eliminate the $f_{i}^{c}$ term. The symmetry and/or skew-symmetry of $f_{i}^{a}$ and $f_{i}^{b}$ can also be used to directly eliminate $K_{I}$ or $K_{I I}$ when solving for the other. The choice of the four displacement components in obtaining equations (7) to (10), namely $u_{r}^{A}=u_{r}\left(l_{E} / 4, \pi\right), u_{r}^{B}=u_{r}\left(l_{E}, \pi\right), u_{\theta}^{A}=u_{\theta}\left(l_{E} / 4, \pi\right)$, and $u_{\theta}^{B}=u_{\theta}\left(l_{E} / 4, \pi\right)$ allows this approach. $r_{i}=l_{E} / 4$ and $r_{i}=l_{E}$ are used for convenience to exploit nodal displacements in the quarter-point elements. However, displacements at other points (not necessarily nodes) can be used instead to solve equation (13).

Through this generalization of the original displacement correlation method, the special quarterpoint element and near-tip region mesh refinement can be eliminated, and we can substitute the displacements at appropriate reference points and other necessary information into equation (13) to solve for SIF's. In the selection of the reference points, we first consider points with $\theta= \pm \pi$, consistent with the original displacement correlation method, where the features of $f_{r}^{a}(\pi)=0$ and $f_{\theta}^{b}(\pi)=0$ simplifies solution. If quadratic elements (i.e. shape functions are second-degree 
polynomials) are used, we can use $r=l_{E} / 2$ and $r=l_{E}$, which are both within the first layer of elements about the crack tip. Appealing to symmetry, we have

$$
\begin{aligned}
& u_{r}\left(l_{E} / 2, \pi\right)-u_{r}\left(l_{E} / 2,-\pi\right)=\sqrt{2 l_{E}} f_{r}^{b}(\pi) K_{I I}+\frac{l_{E}}{2}\left[f_{r}^{c}(\pi)-f_{r}^{c}(-\pi)\right] \\
& u_{r}\left(l_{E}, \pi\right)-u_{r}\left(l_{E},-\pi\right)=2 \sqrt{l_{E}} f_{r}^{b}(\pi) K_{I I}+l_{E}\left[f_{r}^{c}(\pi)-f_{r}^{c}(-\pi)\right] \\
& u_{\theta}\left(l_{E} / 2, \pi\right)-u_{\theta}\left(l_{E} / 2,-\pi\right)=\sqrt{2 l_{E}} f_{\theta}^{a}(\pi) K_{I}+\frac{l_{E}}{2}\left[f_{\theta}^{c}(\pi)-f_{\theta}^{c}(-\pi)\right] \\
& u_{\theta}\left(l_{E}, \pi\right)-u_{\theta}\left(l_{E},-\pi\right)=2 \sqrt{l_{E}} f_{\theta}^{a}(\pi) K_{I}+l_{E}\left[f_{\theta}^{c}(\pi)-f_{\theta}^{c}(-\pi)\right]
\end{aligned}
$$

Solving the above equations yield the formulation for the generalized displacement correlation (GDC) method as

$$
\begin{aligned}
& K_{I}=\frac{2 u_{\theta}\left(l_{E} / 2, \pi\right)-2 u_{\theta}\left(l_{E} / 2,-\pi\right)-u_{\theta}\left(l_{E}, \pi\right)+u_{\theta}\left(l_{E},-\pi\right)}{(2 \sqrt{2}-2) \sqrt{l_{E}} f_{\theta}^{a}(\pi)} \\
& K_{I I}=\frac{2 u_{r}\left(l_{E} / 2, \pi\right)-2 u_{r}\left(l_{E} / 2,-\pi\right)-u_{r}\left(l_{E}, \pi\right)+u_{r}\left(l_{E},-\pi\right)}{(2 \sqrt{2}-2) \sqrt{l_{E}} f_{r}^{b}(\pi)}
\end{aligned}
$$

where the constants $f_{\theta}^{a}(\pi)=f_{r}^{b}(\pi)=-\beta / \sqrt{2 \pi} G$ follow from equations (1) to (3). This set of equations does not require quarter-point elements around the crack tip, but does require quadratic elements (6-node triangle or 8-node quadrilateral in 2D). Since the objective of this paper is to generalize the displacement correlation method, we further consider finite element models where linear elements (3-node triangle or 4-node quadrilateral) are used. Under this condition, equations (15) and (16) result in zero SIF's owing to the linear shape functions. Using displacements across two layers of elements around the tip (i.e. at $r=l_{E}$ and $r=2 l_{E}$ ) and replacing $l_{E} / 2$ in the above equations with $l_{E}$ and $l_{E}$ with $2 l_{E}$ solve this problem, but renders the method impractical for modeling fractures with arbitrary paths. Figure 3 shows two problematic scenarios commonly addressed through FEM modeling of fractures: (a) sawtooth-shaped fractures typical in perturbed meshes where minor perturbation to node locations in the undeformed mesh is adopted to introduce randomness into fracture paths, and (b) a fracture having changed the direction of propagation. In both scenarios, the locations of points $\left(2 l_{E}, \pi\right)$ and $\left(2 l_{E},-\pi\right)$ are ambiguous, making the method above inapplicable. To address this, we use displacements of points with $\theta=-\pi / 2,0$, and $\pi / 2$ and $r=l_{E}$ and $r=2 l_{E}$, and also exploit the symmetry of $f_{r}^{a}$ and $f_{\theta}^{b}$ and skew-symmetry of $f_{\theta}^{a}$ and $f_{r}^{b}$ to obtain

$$
\begin{aligned}
& u_{r}\left(l_{E}, \pi / 2\right)+u_{r}\left(l_{E},-\pi / 2\right)=2 \sqrt{l_{E}} f_{r}^{a}(\pi / 2) K_{I}+l_{E}\left[f_{r}^{c}(\pi / 2)+f_{r}^{c}(-\pi / 2)\right] \\
& u_{r}\left(2 l_{E}, \pi / 2\right)+u_{r}\left(2 l_{E},-\pi / 2\right)=2 \sqrt{2 l_{E}} f_{r}^{a}(\pi / 2) K_{I}+2 l_{E}\left[f_{r}^{c}(\pi / 2)+f_{r}^{c}(-\pi / 2)\right]
\end{aligned}
$$


$u_{\theta}\left(l_{E}, 0\right)=\sqrt{l_{E}} f_{\theta}^{b}(0) K_{I I}+l_{E} f_{\theta}^{c}(0)$

$u_{\theta}\left(2 l_{E}, 0\right)=\sqrt{2 l_{E}} f_{\theta}^{b}(0) K_{I I}+2 l_{E} f_{\theta}^{c}(0)$

which yield

$$
\begin{aligned}
& K_{I}=\frac{2 u_{r}\left(l_{E}, \pi / 2\right)+2 u_{r}\left(l_{E},-\pi / 2\right)-u_{r}\left(2 l_{E}, \pi / 2\right)-u_{r}\left(2 l_{E},-\pi / 2\right)}{(4-2 \sqrt{2}) \sqrt{l_{E}} f_{r}^{a}(\pi / 2)} \\
& K_{I I}=\frac{2 u_{\theta}\left(l_{E}, 0\right)-u_{\theta}\left(2 l_{E}, 0\right)}{(2-\sqrt{2}) \sqrt{l_{E}} f_{\theta}^{b}(0)}
\end{aligned}
$$

where the constants $f_{r}^{a}(\pi / 2)=(2 \beta-1) / 4 \sqrt{\pi} G$ and $f_{\theta}^{b}(0)=(1-\beta) / \sqrt{2 \pi} G$. We term the GDC method based on equations (15) and (16) "Method A", and that based on equations (18) and (19) "Method B". Method B can be applied to any finite element types, and is therefore "more general" than Method A. Method A only requires displacements across one layer of elements around the tip while Method B requires two layers. Neither Method A nor Method B requires a special meshing scheme at the near-tip region, such as a mesh type or mesh resolution different from that of the remainder of the computation domain. Both methods are easy to implement in existing FEM packages. Note that the points where displacements are used in the calculation need not to be nodes of the finite element mesh.

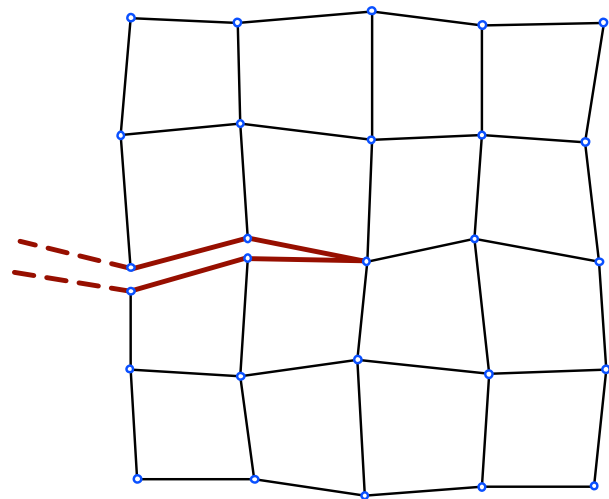

(a)

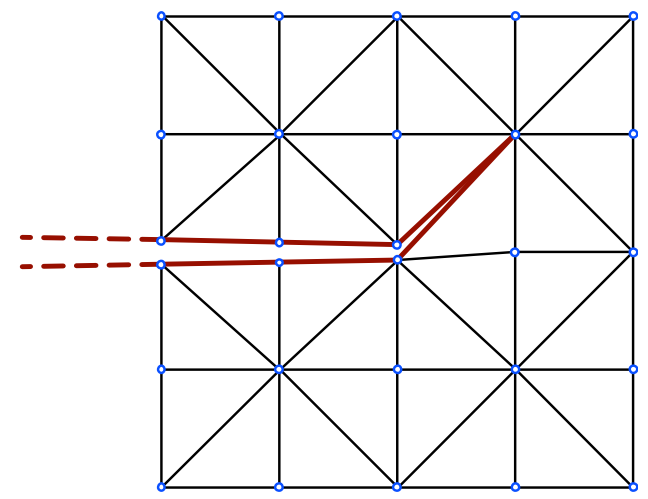

(b)

Figure 3 Two common scenarios where the locations of points $\left(2 l_{E}, \pi\right)$ and $\left(2 l_{E},-\pi\right)$ are ambiguous.

\section{Enhancement of the generalized method}

Error in the calculated stress intensity factors using the GDC method can be attributed to at least two sources: 
1) The inability of the adopted finite element's shape functions to accurately represent the neartip displacement field. The quarter-point element family was originally formulated for the very purpose of better representing the near-tip field by including a square-root term in the shape functions in the ray directions.

2) Omission of higher-order terms (1) and (3). These equations are accurate at the near-tip region, where the distances to the fracture tip and other sources inducing high displacement gradient are much smaller than the length of the fracture itself. In the GDC method, displacements at distances $l_{E}$ and $2 l_{E}$ (or $l_{E} / 2$ and $l_{E}$ ) are used. Therefore, error increases with the ratio of element size to the fracture length.

In order to demonstrate the accuracy of the GDC method, we use the proposed method on the simplest fracture system, i.e. a finite-length fracture in an infinite domain as shown in Figure 4. The fracture system considered here is straight crack of length $2 a$ in a 2D infinite medium. Since most FEM models can accurately represent the linear displacement field induced by the loading condition in Figure 1(c), only the loading conditions in Figure 1(a) and (b) are combined and modeled. However, the effects of homogeneous stress fields are appropriately handled in the formulations of the GDC method, and the superposition of such a field would not affect the calculated SIF's. The near-tip mesh configuration can have a considerable effect on the accuracy of the original displacement-based methods (e.g. [22]); in all the numerical examples in the current and next section, the mesh configuration shown in Figure 5(a) is used, and fracture tips are located at nodes shared by eight triangular elements. The other mesh configurations shown in Figure 5 will be investigated in section 6 . In linearly elastic problems, the shear modulus of the medium, $G$ does not affect the calculated stress intensity factors and thus can be arbitrarily selected. The model is assumed to be a plain-stress problem with a Poisson's ratio of 0.2. The effects of the Poisson's ratio will also be discussed in section 6 . The finite element mesh is sufficiently large (with each dimension longer than 100a) such that the effects of the finite boundaries are minimal and the domain can be considered infinite. We use quadratic (6-node) triangle elements with full-integration (three Gaussian points) for both Methods A and B in this study, although Method B is not restricted to quadratic elements. 


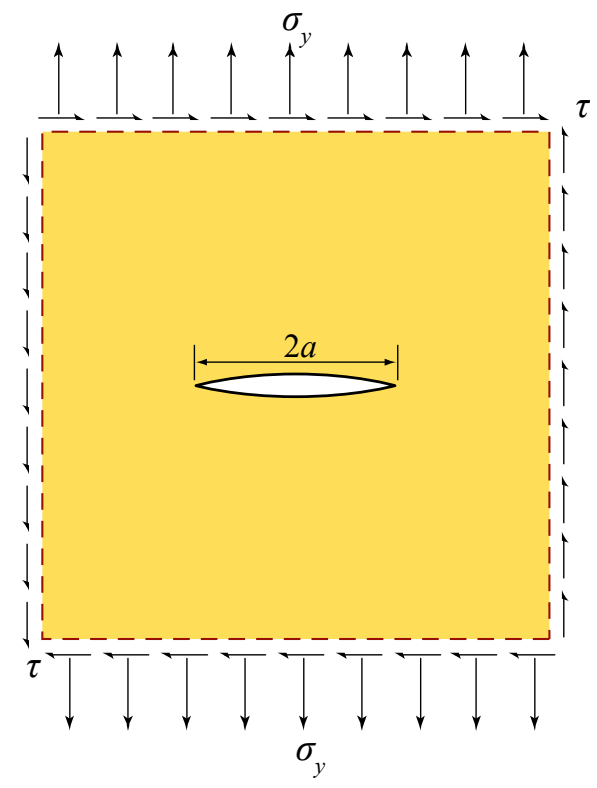

Figure 4 A finite-length crack in an infinite medium.

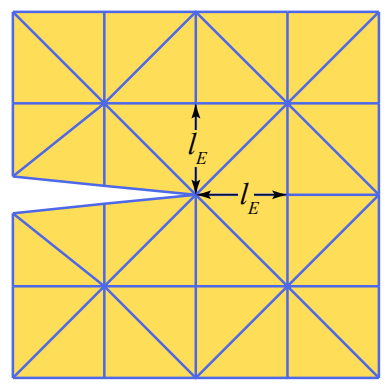

(a) Mesh configuration $\mathrm{i}$

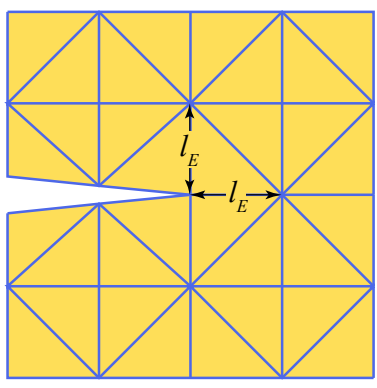

(b) mesh-ii

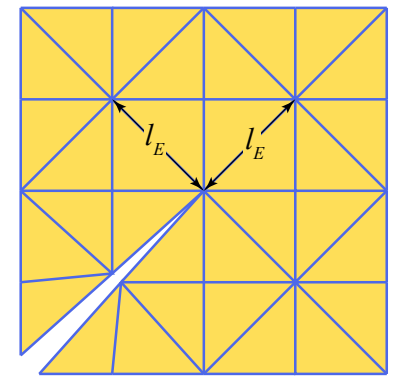

(b) mesh-iii

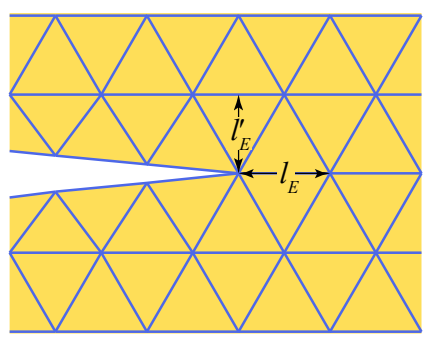

(b) mesh-iv

Figure 5 Four mesh configurations considered in this study. The conventional six-node triangle element is used in all the numerical examples of the present study but the mid-edge node is not shown in this figure.

The theoretical solutions for the stress intensity factors in this crack configuration are $K_{I}=\sqrt{\pi a} \sigma_{y}$ and $K_{I I}=\sqrt{\pi a} \tau$. Numerical solutions of the SIF's, denoted by $K_{I}^{\prime}$ and $K_{I I}^{\prime}$ are obtained by solving finite element models with various levels of mesh resolutions (quantified by $a / l_{E}$, the ratio of the half crack length to element length) and substituing the obtained displacement values into equations (15) and (16) or (18) and (19). We then seek an enhancement measure in the form of a "correction multiplier" to be added to equations (15), (16), (18), and (19). We will test the performance of the corrected/enhanced formulation on a number of more complex crack systems in next section for Methods A and B. The values of $C_{I}=K_{I} / K_{I}^{\prime}$ and $C_{I I}=K_{I I} / K_{I I}^{\prime}$, which are the multipliers that need to be applied to equations (15) and (16) or (18) and (19), respectively to correct the numerical solutions are shown in Figure 6 as functions of $a / l_{E}$. The correction factors are significantly larger than unity, since the 6-node triangular finite 
element cannot accurately represent the near-tip displacement field. $C_{I}$ and $C_{I I}$ both converge to constant values as the element size becomes smaller relative to the crack length. We can fit the discrete data points with the following empirical relationship

$$
C=\frac{\alpha_{1}}{\sqrt{1-\alpha_{2} l_{E} / a}}
$$

which has a similar format as the correction term used in [23]. The regression results are

$$
\begin{aligned}
& C_{I}^{A}=\frac{1.555}{\sqrt{1+0.640 l_{E} / a}} \\
& C_{I I}^{A}=\frac{2.831}{\sqrt{1+1.163 l_{E} / a}} \\
& C_{I}^{B}=\frac{1.260}{\sqrt{1-0.138 l_{E} / a}} \\
& C_{I I}^{B}=\frac{1.727}{\sqrt{1-0.845 l_{E} / a}}
\end{aligned}
$$

where the superscripts ${ }^{A}$ and ${ }^{B}$ of $C_{I}$ and $C_{I I}$ indicate whether the correction multipliers are for Method A or Method B. The coefficients of determination $\left(R^{2}\right)$ for all regressions are greater than 0.99 .

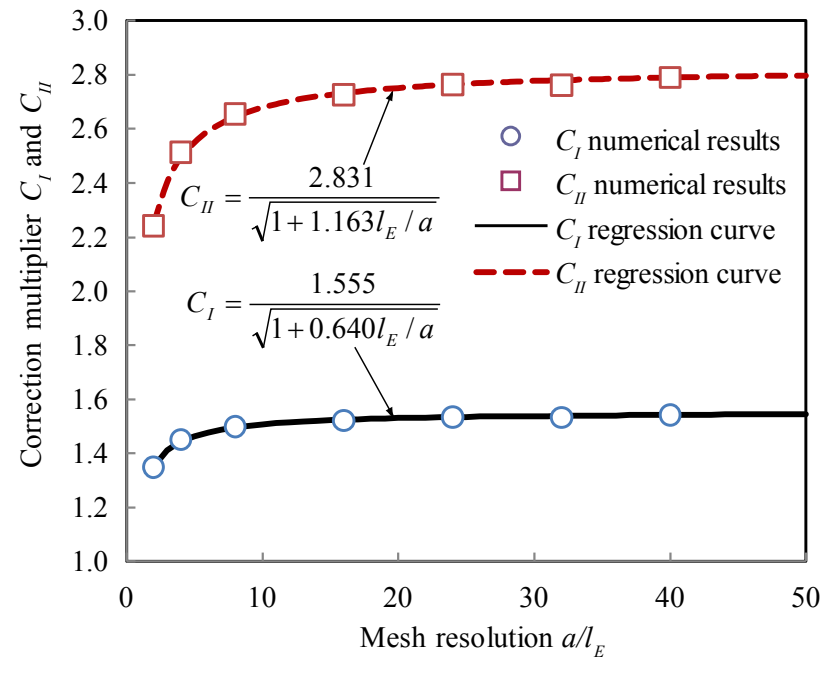

(a)

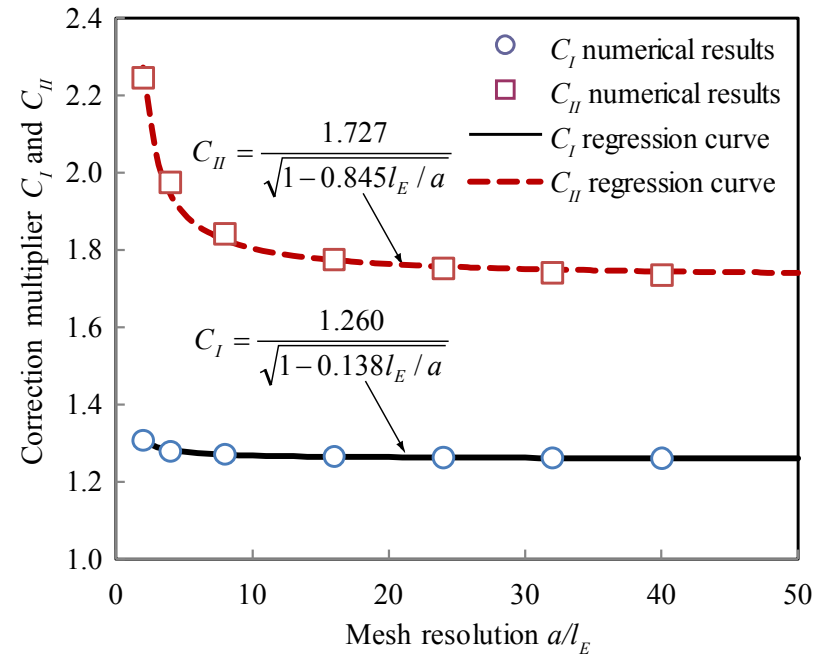

(b)

Figure 6 The effects of the mesh resolution on the correction multipliers. (a) Results for Method A; (b) results for Method B.

The correction multipliers calculated using equation (21) converge but not to unity. This appears counterintuitive because even though the shape functions (quadratic for the above calculations and linear if linear elements were used) of a single element does not accommodate the square 
root terms in equation (6), refining the mesh (with smaller $l_{E}$ ) should result in piecewise quadratic shape functions for the mesh as a whole better representing the displacement field. However, regardless of the refinement level, only displacements within the first one (Method A) or two (Method B) layers of elements around the fracture tip are used. As the mesh is refined, the reference points where displacement information is used in the calculation are closer to the fracture tip. For infinitesimal elements, this mechanism can eliminate the error induced by the second source of error, but not the first. A similar phenomenon exist for the original displacement-based methods: Numerous studies have observed that errors of these methods do not converge to zero as the near-tip mesh is refined $[12,13,18,19,22]$ and an explanation was offered by Harrop [24].

\section{Accuracy of the generalized method for different fracture configurations}

The values as well as the regression formula of the correction multipliers in section 4 are obtained for a specific fracture-load configuration. Considering that the main purpose of this correction term is to correct errors caused by the finite elements' inability to accurately represent the near-tip displacement field described by equations (1) to (3), we hypothesize that the same multipliers can be applied to all other crack-load configurations and obtain reasonable SIF results. In this section, we apply the correction multipliers obtained from the special case in section 4 to a spectrum of fracture configurations to test this hypothesis. Special attention is paid to coarse meshes and effects of interference between neighboring fractures and between fractures and free surfaces. Achieving acceptable accuracy under these conditions is crucial for managing the computational cost of the simulation of dynamic fracture propagation in complex fracture systems. Both Method A and Method B are evaluated for the first case in section 5.1. Since the mathematical and mechanical principles behind these two methods are similar, only the more general Method B is considered for the other three fracture-load configurations.

\subsection{Center-cracked infinite strip with a finite width}

Consider a center-cracked strip with an infinite length but finite width $2 b$. The crack is $2 a$ long and perpendicular to the longitudinal direction of the strip as shown in Figure 7(a). The strip is subjected to a tensile stress $\sigma$ in the longitudinal direction and a uniformly distributed shear stress $\tau$ along the fracture faces, inducing mode-I and mode-II stress concentration, respectively. The stress intensity factors are

$$
K_{I}=\sigma \sqrt{\pi a} F_{I}(a / b) \text { and } K_{I I}=\tau \sqrt{\pi a} F_{I I}(a / b)
$$

where $F_{I}$ and $F_{I I}$ are the fracture-configuration correction factors that can be estimated using the modified Koiter's formula [1]: 
$F_{I}(a / b)=F_{I I}(a / b)=\left[1-0.025(a / b)^{2}+0.06(a / b)\right]\left(\cos \frac{\pi a}{2 b}\right)^{-1 / 2}$

with a relative error of less than $0.1 \%$ for any $a / b$ value. In this and other examples, if $F_{I}$ and $F_{I I}$ are close to unity, it means this fracture-load configuration is similar to the reference configuration of a single fracture in an infinite plane.

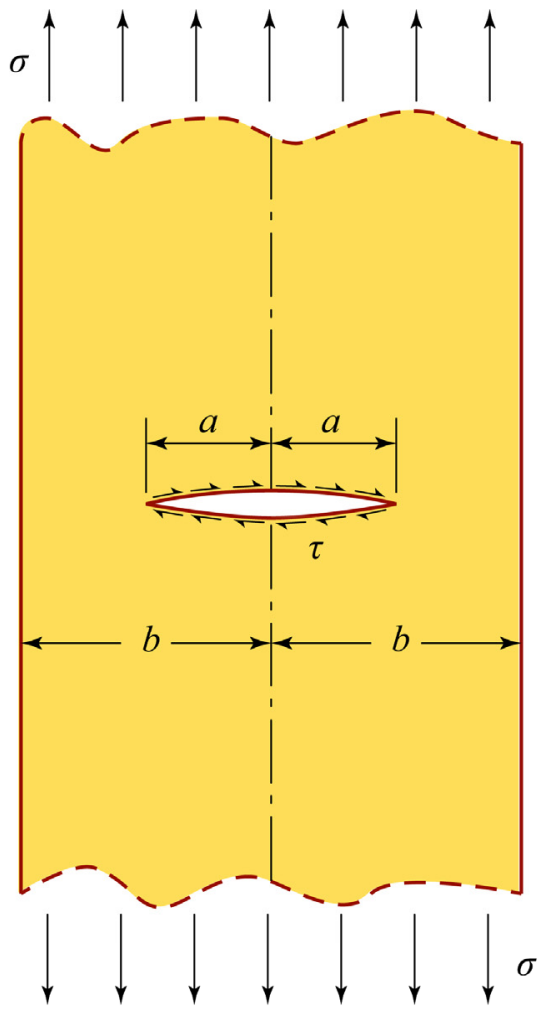

(a)

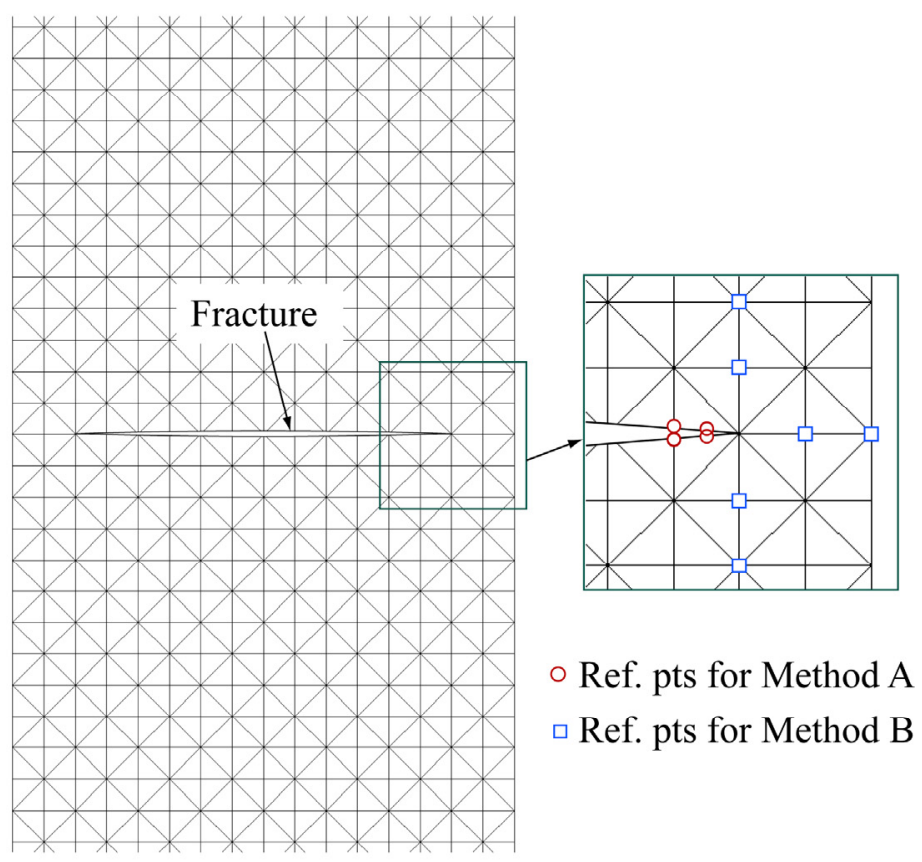

(b)

Figure 7 Center-cracked infinite strip with a finite width. (a) The crack configuration; (b) the mesh for the case where $b=8 l_{E}$ and $a / b=0.75$ (with opening of the fracture exaggerated). The reference points used by Method A and Method B are indicated in the figure.

To apply the GDC method, the strip is discretized into a finite element mesh of a length that is more than 12 times longer than its width, which is found to sufficiently approximate the infinite length according to a sensitivity analysis. Different levels of mesh refinement with $b / l_{E}$ ranging from 4 to 64 as well as various crack length-to-strip width ratios, i.e., $a / b=0.125,0.25,0.50$, 0.75 , and 0.875 are adopted to investigate the effects of these two factors. Due to the symmetry of the crack and mesh configuration, the tensile stress $\sigma$ does not contribute to the calculated $K_{I I}$ and $\tau$ does not contribute to $K_{I}$. In all the numerical examples in section 4, a Poisson's ratio of 0.2 and the crack tip mesh configuration shown in Figure 5(a) (eight triangle elements connected to the tip) are used. The effects of the Poisson's ratio and crack tip mesh configuration will be 
studied in section 6. To allow precise comparison, the calculation results of the GDC method (both Method A and Method B) with the correction multipliers computed using equation (21) applied, as well as the theoretical solution based on equation (23) are shown in Tables 1-A to 2B. Note that the values of $F_{I}$ and $F_{I I}$, instead of the stress intensity factors $K_{I}$ and $K_{I I}$ are shown. $F_{I}$ and $F_{I I}$ can be considered normalized values of the SIF's. Due to the relationships described in equation (22), the relatively errors for $K_{I}$ and $K_{I I}$ are the same as those for $F_{I}$ and $F_{I I}$, respectively.

Table 1-A Calculated $F_{I}$ values using the GDC method (Method A) for the center-cracked infinite strip.

\begin{tabular}{cccccccccccc}
\hline \multicolumn{1}{c}{$F_{I}$, numerical result } & \multicolumn{9}{c}{ Relative error (\%) } & \multicolumn{2}{c}{$F_{I}(a / b)$} \\
& $b / l_{E}=4$ & 8 & 16 & 32 & 64 & $b / l_{E}=4$ & 8 & 16 & 32 & 64 & eq. $(23)$ \\
\hline 0.125 & $\mathrm{~N}^{\mathrm{a}} \mathrm{A}^{\mathrm{a}}$ & $\mathrm{N} / \mathrm{A}^{\mathrm{a}}$ & 1.011 & 1.004 & 1.007 & $\mathrm{~N} / \mathrm{A}^{\mathrm{a}}$ & $\mathrm{N} / \mathrm{A}^{\mathrm{a}}$ & 0.1 & -0.6 & -0.2 & 1.009 \\
0.25 & $\mathrm{~N} / \mathrm{A}^{\mathrm{a}}$ & 1.038 & 1.032 & 1.036 & 1.040 & $\mathrm{~N} / \mathrm{A}^{\mathrm{a}}$ & -0.1 & -0.7 & -0.3 & 0.1 & 1.039 \\
0.50 & 1.168 & 1.171 & 1.179 & 1.186 & 1.189 & -1.5 & -1.3 & -0.6 & 0.0 & 0.3 & 1.186 \\
0.75 & $\mathrm{~N} / \mathrm{A}^{\mathrm{a}}$ & 1.595 & 1.612 & 1.622 & 1.628 & $\mathrm{~N} / \mathrm{A}^{\mathrm{a}}$ & -1.8 & -0.8 & -0.1 & 0.2 & 1.624 \\
0.875 & $\mathrm{~N} / \mathrm{A}^{\mathrm{a}}$ & $\mathrm{N} / \mathrm{A}^{\mathrm{a}}$ & 2.271 & 2.288 & 2.300 & $\mathrm{~N} / \mathrm{A}^{\mathrm{a}}$ & $\mathrm{N} / \mathrm{A}^{\mathrm{a}}$ & -1.3 & -0.5 & 0.0 & 2.300 \\
\hline
\end{tabular}

Note: ${ }^{\mathrm{a}} \mathrm{N} / \mathrm{A}$, numerical results unavailable due to the incompatibility between the $a / b$ value and the mesh configuration.

Table 1-B Calculated $F_{I}$ values using the GDC method (Method B) for the center-cracked infinite strip.

\begin{tabular}{cccccccccccc}
\hline \multicolumn{1}{c}{$F_{I}$, numerical result } & \multicolumn{7}{c}{ Relative error $(\%)$} & & $F_{I}(a / b)$ \\
& $b / l_{E}=4$ & 8 & 16 & 32 & 64 & $b / l_{E}=4$ & 8 & 16 & 32 & 64 & eq. $(23)$ \\
\hline 0.125 & N/A & N/A & 1.008 & 1.011 & 1.009 & N/A & N/A & -0.1 & 0.2 & 0.0 & 1.009 \\
0.25 & N/A & 1.036 & 1.040 & 1.038 & 1.037 & N/A & -0.3 & 0.1 & -0.1 & -0.1 & 1.039 \\
0.50 & 1.196 & 1.186 & 1.182 & 1.183 & 1.184 & 0.8 & 0.0 & -0.4 & -0.3 & -0.2 & 1.186 \\
0.75 & N/A & 1.640 & 1.618 & 1.617 & 1.619 & N/A & 1.0 & -0.4 & -0.5 & -0.3 & 1.624 \\
0.875 & N/A & N/A & 2.325 & 2.295 & 2.291 & N/A & N/A & 1.1 & -0.2 & -0.4 & 2.300 \\
\hline
\end{tabular}


Table 2-A Calculated $F_{I I}$ values using the GDC method (Method A) for the center-cracked infinite strip.

\begin{tabular}{cccccccccccc}
\hline \multicolumn{1}{c}{$a / b$} & \multicolumn{4}{c}{$F_{I I}$, numerical result } & \multicolumn{3}{c}{ Relative error (\%) } & \multicolumn{2}{c}{$F_{I I}(a / b)$} \\
& $b / l_{E}=4$ & 8 & 16 & 32 & 64 & $b / l_{E}=4$ & 8 & 16 & 32 & 64 & eq.(23) \\
\hline 0.125 & N/A & N/A & 1.013 & 1.000 & 1.006 & N/A & N/A & 0.3 & -0.9 & -0.3 & 1.009 \\
0.25 & N/A & 1.040 & 1.030 & 1.038 & 1.045 & N/A & 0.1 & -0.8 & -0.1 & 0.6 & 1.039 \\
0.50 & 1.165 & 1.172 & 1.188 & 1.201 & 1.208 & -1.8 & -1.2 & 0.2 & 1.2 & 1.8 & 1.186 \\
0.75 & N/A & 1.579 & 1.621 & 1.645 & 1.658 & N/A & -2.8 & -0.2 & 1.3 & 2.1 & 1.624 \\
0.875 & N/A & N/A & 2.241 & 2.294 & 2.323 & N/A & N/A & -2.6 & -0.3 & 1.0 & 2.300 \\
\hline
\end{tabular}

Table 2-B Calculated $F_{I I}$ values using the GDC method (Method B) for the center-cracked infinite strip.

\begin{tabular}{|c|c|c|c|c|c|c|c|c|c|c|c|}
\hline \multirow{2}{*}{$a / b$} & \multicolumn{5}{|c|}{$F_{I I}$, numerical result } & \multicolumn{5}{|c|}{ Relative error (\%) } & \multirow{2}{*}{$\begin{array}{l}F_{I I}(a / b) \\
\text { eq. }(23)\end{array}$} \\
\hline & $b / l_{E}=4$ & 8 & 16 & 32 & 64 & $b / l_{E}=4$ & 8 & 16 & 32 & 64 & \\
\hline 0.125 & N/A & N/A & 1.021 & 0.994 & 1.001 & N/A & N/A & 1.1 & -1.6 & -0.8 & 1.009 \\
\hline 0.25 & N/A & 1.027 & 1.018 & 1.031 & 1.041 & N/A & -1.2 & -2.0 & -0.8 & 0.2 & 1.039 \\
\hline 0.50 & $0.014^{b}$ & 0.972 & 1.132 & 1.181 & 1.200 & -98.8 & -18.1 & -4.5 & -0.4 & 1.2 & 1.186 \\
\hline 0.75 & N/A & $-0.841^{b}$ & 1.124 & 1.502 & 1.610 & N/A & -152 & -30.8 & -7.6 & -0.9 & 1.624 \\
\hline 0.875 & N/A & $\mathrm{N} / \mathrm{A}^{\mathrm{a}}$ & $-1.710^{b}$ & 1.432 & 2.070 & N/A & N/A & -174 & -37.8 & -10.0 & 2.300 \\
\hline
\end{tabular}

Note: ${ }^{b}$ degenerate results; see discussion below. The Bold typeface used in other tables highlights degenerate results owing to similar reasons.

The results show that Method B for mode-I fracturing and Method A for both mode-I and -II are fairly accurate for all the scenarios considered, including those with very coarse meshes. The relative errors are generally smaller than $2 \%$ with few exceptions. The accuracy of Method-B for mode-II fracturing seems to be dependent on the fracture geometry and mesh resolution. For $b / l_{E}=4$ with $a / b=0.5 ; b / l_{E}=8$ with $a / b=0.75$; and $b / l_{E}=16$ with $a / b=0.875$ ), erroneous results are obtained. In these three situations, the fracture tip is two elements away (i.e. $\left.(b-a) / l_{E}=2\right)$ from the lateral boundary. One of the displacement components used in equation $(19), u_{\theta}\left(2 l_{E}, 0\right)$ happens to be at the lateral boundary. The mechanical response at this point is substantially affected by the free-surface boundary condition and violate an assumption of the GDC method. This is not an issue for Method A or the calculation of $K_{I}$ using Method B because none of the displacement components used in equations (15), (16), and (18) is at the boundary. At the same mesh refinement level, if the distance between the crack tip and the lateral free-surface boundary is $4 l_{E}$ 
instead of $2 l_{E}$, the relative error for $K_{I I}$ (Method B) is approximately between $20 \%$ and $40 \%$, which though suboptimal for typical mechanical engineering applications is often acceptable for geo-science or geo-engineering scenarios due to the high aleatoric uncertainty in geo-systems. Nevertheless, if the crack tip is $6 l_{E}$ or farther away from the free surface, the error drops below $10 \%$ for $K_{I I}$ by Method B.

5.2 Three-point bending beam with a notch at mid-span

Consider a beam specimen with a span-to-height ratio of $s / b=4$ with a notch of length $a$ cut at the mid-span as shown in Figure 8. The beam is subjected to a mid-span force $P$. Due to the symmetry of the configuration, the mode-II stress intensity factor is zero, and for mode-I

$$
K_{I}=\frac{3 P s}{2 b^{2}} \sqrt{\pi a} F_{I}(a / b)
$$

where $F_{I}(a / b)$ is the fracture-configuration correction factor, with similar meaning to its counterpart in equation (22) but different values. Its value can be calculated using the following dimensionless regression equation proposed by Srawley [25] with a relative error smaller than $0.5 \%$

$$
F(a / b)=\frac{1.99-a / b(1-a / b)\left[2.15-3.93 a / b+2.7(a / b)^{2}\right]}{(1+2 a / b)(1-a / b)^{3 / 2} \sqrt{\pi}}
$$

To test the accuracy of the GDC method on this configuration, we perform FEM analysis with different levels of mesh refinement and different notch lengths. The results of Method-B are summarized in Table 3 in a manner similar to that of Tables 1 and 2. The results are generally accurate. In the worst case scenario, where the height direction of the beam is discretized into four element, the relative error is $11.7 \%$, which remains acceptable for many engineering applications. As the mesh is refined, the numerical results for each geometrical configuration generally converge to the closed-form solution with some minor fluctuation (a few tenths of a percent), which is within the $0.5 \%$ error inherent in the closed-form solution. The accuracy is compromised when the notch is short or long compared with the beam height (e.g. $a / b=0.125$ or 0.875). In both cases, the points where the displacements are used in the GDC method have similar distances to the notch tip and to the lower or upper free surface of the beam and are not within the near-tip region. 


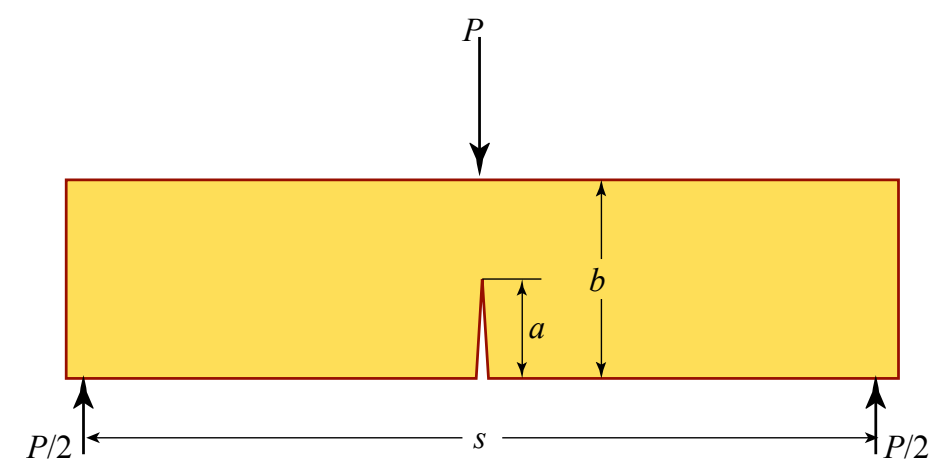

Figure 8 Three-point bending beam with a mid-span notch.

Table 3 Calculated $F_{I}$ values using the GDC method for the three-point bend beam (Method B only).

\begin{tabular}{cccccccccccc}
\hline \multicolumn{1}{c}{$a / b$} & \multicolumn{4}{c}{$F_{I}$, numerical result } & \multicolumn{5}{c}{ Relative error (\%) } & \multicolumn{2}{c}{$F_{I}(a / b)$} \\
& $b / l_{E}=4$ & 8 & 16 & 32 & 64 & $b / l_{E}=4$ & 8 & 16 & 32 & 64 & eq. (25) \\
\hline 0.125 & N/A & N/A & 0.944 & 0.965 & 0.972 & N/A & N/A & -5.1 & -3.0 & -2.3 & 0.995 \\
0.25 & N/A & 1.013 & 1.005 & 1.003 & 1.001 & N/A & 0.5 & -0.2 & -0.4 & -0.6 & 1.007 \\
0.50 & 1.581 & 1.468 & 1.422 & 1.409 & 1.406 & 11.7 & 3.7 & 0.4 & -0.5 & -0.7 & 1.416 \\
0.75 & N/A & 3.623 & 3.439 & 3.369 & 3.352 & N/A & 8.2 & 2.7 & 0.6 & 0.1 & 3.349 \\
0.875 & N/A & N/A & 9.469 & 9.075 & 8.929 & N/A & N/A & 7.1 & 2.6 & 1.0 & 8.843 \\
\hline
\end{tabular}

\subsection{Two finite-length fractures along a single line}

In sections 5.3 and 5.4, we investigate the accuracy of the GDC method for scenarios with multiple fractures interacting with each other. We first consider the configuration shown in Figure 9, where two finite-length fractures along a single line existing in an infinite plane. This configuration tends to strengthen the stress intensity at the two tips $A$ and $B$, compared with the configurations whether the two cracks exist alone in infinite planes. For any tip under a given far-field stress condition ( $\sigma$ and $\tau$ ), the stress intensity factors (mode-I and mode-II) are dependent on certain geometrical features of the system, and the following closed-form solutions are available [1]

$$
\begin{aligned}
& K_{I}^{A}=\sigma \sqrt{\pi b} F_{I}^{A}(a / b, c / b) \\
& K_{I I}^{A}=\tau \sqrt{\pi b} F_{I I}^{A}(a / b, c / b) \\
& K_{I}^{B}=\sigma \sqrt{\pi a} F_{I}^{B}(a / b, c / b)
\end{aligned}
$$

and $K_{I I}^{B}=\tau \sqrt{\pi a} F_{I I}^{B}(a / b, c / b)$ 
where

$$
\begin{aligned}
& F_{I}^{A}=F_{I I}^{A}=\frac{1}{\sqrt{1-\alpha_{A}}}\left[1-\frac{1}{\alpha_{B}}\left\{1-\frac{E(k)}{K(k)}\right\}\right] \\
& F_{I}^{B}=F_{I I}^{B}=\frac{1}{\sqrt{1-\alpha_{B}}}\left[1-\frac{1}{\alpha_{A}}\left\{1-\frac{E(k)}{K(k)}\right\}\right]
\end{aligned}
$$

with $\alpha_{A}=a /(a+c), \alpha_{B}=b /(b+c)$, and $k=\sqrt{\alpha_{A} \alpha_{B}}$ and

$$
\begin{aligned}
& K(k)=\int_{0}^{\pi / 2}\left(1-k^{2} \sin ^{2} \varphi\right)^{-1 / 2} d \varphi \\
& E(k)=\int_{0}^{\pi / 2}\left(1-k^{2} \sin ^{2} \varphi\right)^{1 / 2} d \varphi
\end{aligned}
$$

In the numerical solutions, we fix the length ratio of the two fractures to be $a / b=0.5$ and investigate the effects of the mesh refinement levels $\left(b / l_{E}=4,8\right.$, and 16) and the distance between the two fracture tips $(c / b=1 / 2,1 / 4,1 / 8$, and $1 / 16$ whenever applicable). The finite element model is more than $50 \mathrm{~b}$ long in each dimension to minimize the boundary effects. The numerical results for the two crack tips $A$ and $B$ are summarized in Table 4 and Table 5, respectively.

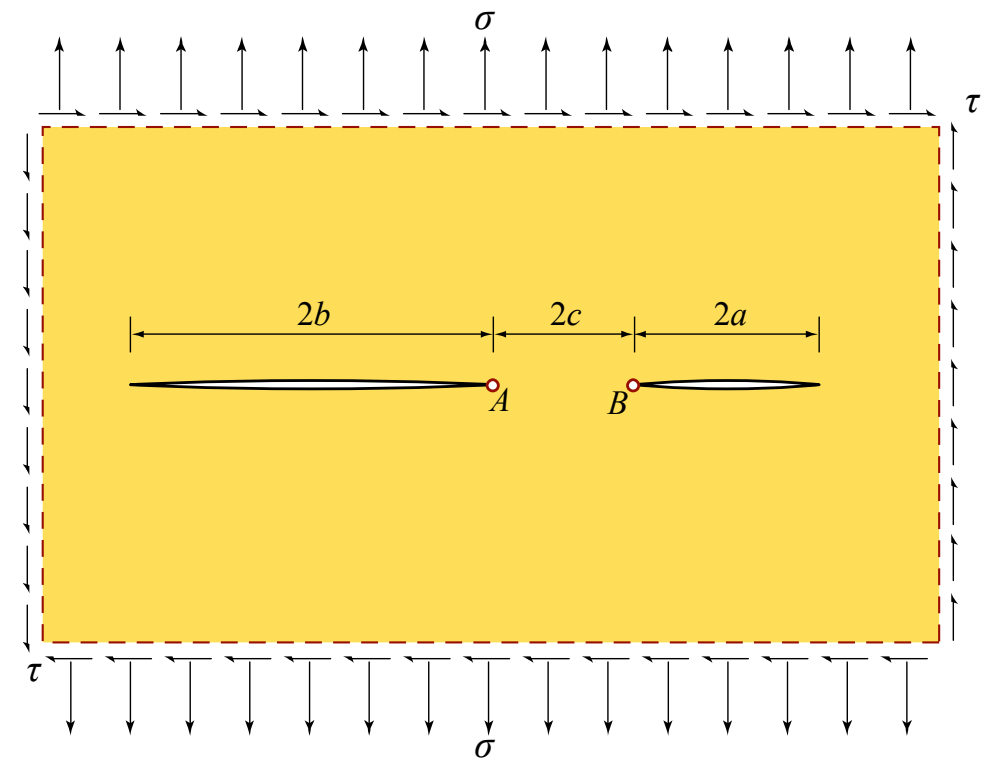

Figure 9 Two finite-length fractures along a single line in an infinite plane. 
Table 4 Calculated stress intensity for the two-fracture case at crack tip $A$ (Method B only).

\begin{tabular}{|c|c|c|c|c|c|c|c|c|c|c|c|c|c|}
\hline \multirow{2}{*}{$b / c$} & \multicolumn{3}{|c|}{$F_{I}$, numerical result } & \multicolumn{3}{|c|}{$F_{I}$, relative error $(\%)$} & \multicolumn{3}{|c|}{$F_{I I}$, numerical result } & \multicolumn{3}{|c|}{$F_{I I}$, relative error $(\%)$} & \multirow{2}{*}{$\begin{array}{c}F_{I}, F_{I I} \\
\text { anly. } \\
\text { solu. }\end{array}$} \\
\hline & $b / l_{E}=4$ & 8 & 16 & $b / l_{E}=4$ & 8 & 16 & $b / l_{E}=4$ & 8 & 16 & $b / l_{E}=4$ & 8 & 16 & \\
\hline 2 & 1.027 & 1.036 & 1.041 & -1.5 & -0.6 & -0.2 & 0.975 & 1.015 & 1.035 & -6.5 & -2.7 & -0.7 & 1.043 \\
\hline 4 & 1.044 & 1.071 & 1.090 & -5.1 & -2.7 & -0.9 & 0.318 & 1.004 & 1.068 & -71.1 & -8.7 & -2.9 & 1.100 \\
\hline 8 & $1.137^{\mathrm{c}}$ & 1.113 & 1.163 & $-5.6^{\mathrm{c}}$ & -7.7 & -3.5 & N/A & 0.246 & 1.076 & N/A & -79.6 & -10.7 & 1.206 \\
\hline 16 & N/A & $1.249^{\mathrm{c}}$ & 1.248 & N/A & $-9.3^{c}$ & -9.3 & N/A & N/A & 0.185 & N/A & N/A & -86.5 & 1.377 \\
\hline 32 & N/A & N/A & $1.445^{\mathrm{c}}$ & N/A & N/A & $-11.4^{\mathrm{c}}$ & N/A & N/A & N/A & N/A & N/A & N/A & 1.632 \\
\hline
\end{tabular}

Table 5 Calculated stress intensity for the two-fracture case at crack tip $B$ (Method B only).

\begin{tabular}{|c|c|c|c|c|c|c|c|c|c|c|c|c|c|}
\hline \multirow{2}{*}{$b / c$} & \multicolumn{3}{|c|}{$F_{I}$, numerical result } & \multicolumn{3}{|c|}{$F_{I}$, relative error $(\%)$} & \multicolumn{3}{|c|}{$F_{I I}$, numerical result } & \multicolumn{3}{|c|}{$F_{I I}$, relative error $(\%)$} & \multirow{2}{*}{$\begin{array}{c}F_{I}, F_{I I} \\
\text { anly. } \\
\text { solu. }\end{array}$} \\
\hline & $b / l_{E}=4$ & 8 & 16 & $b / l_{E}=4$ & 8 & 16 & $b / l_{E}=4$ & 8 & 16 & $b / l_{E}=4$ & 8 & 16 & \\
\hline 2 & 1.078 & 1.113 & 1.122 & -4.2 & -1.1 & -0.3 & 0.978 & 1.058 & 1.098 & -13.1 & -6.0 & -2.5 & 1.126 \\
\hline 4 & 1.117 & 1.197 & 1.238 & -11.2 & -4.8 & -1.5 & -0.526 & 1.038 & 1.177 & -142 & -17.4 & -6.3 & 1.257 \\
\hline 8 & $1.304^{\mathrm{c}}$ & 1.287 & 1.387 & $-10.9^{\mathrm{c}}$ & -12.1 & -5.2 & N/A & -0.370 & 1.204 & N/A & -125 & -17.7 & 1.464 \\
\hline 16 & N/A & $1.533^{\mathrm{c}}$ & 1.541 & N/A & $-12.9^{\mathrm{c}}$ & -12.5 & N/A & N/A & -0.270 & N/A & N/A & -115 & 1.761 \\
\hline 32 & N/A & N/A & $1.878^{\mathrm{c}}$ & N/A & N/A & $-13.4^{\mathrm{c}}$ & N/A & N/A & N/A & $\mathrm{N} / \mathrm{A}$ & N/A & N/A & 2.169 \\
\hline
\end{tabular}

Note: ${ }^{\mathrm{C}}$ limit of the mesh coarseness reached where only one element exist between tip $A$ and tip B. $K_{I I}$ cannot be calculated at this level of mesh refinement using Method B.

The trends observed in this series of results are similar to those from sections 5.1 and 5.2.

Method B of the GDC method is more accurate for mode-I stress intensity than for mode-II. Even under pathological conditions, i.e. mesh coarseness limit reached and strong numerical coupling between the two tips, the error is of the order of $10 \%$. The accuracy for mode-II is nonideal but still acceptable for many applications. The only exceptions are when the two tips are only two elements away from each other. In this situation, $u_{\theta}\left(2 l_{E}, 0\right)$ used in equation (19) for a tip is the displacement of the other tip, resulting in strong numerical coupling between the two fractures. In these situations, Method A is more appropriate since it uses displacements "behind" fracture tips, where less numerical coupling between the two fractures is expected.

5.4 An infinite array of parallel fractures in an infinite plane

Consider the fracture configuration shown in Figure 10 where an infinite array of parallel finitelength cracks are periodically arranged on an infinite plane subjected to far-field stress. The 
interaction between fractures tends to reduce mode-I stress intensity but enhance mode-II stress intensity. The stress intensity factors are $K_{I}=\sigma \sqrt{\pi a} F_{I}(a / h)$ and $K_{I I}=\tau \sqrt{\pi a} F_{I I}(a / h)$ where $F_{I}$ and $F_{I I}$ are the crack configuration correction factors as functions of the crack length and the interval between neighboring cracks. The analytical solutions for $F_{I}$ and $F_{I I}$ are unavailable but wellaccepted numerical solutions are presented in [1] and are plotted as continuous curves in Figure 11. In the FEM solution of this study, we investigate the effects of mesh refinement level $\left(a / l_{E}=16,8,4\right.$, and 2$)$ and distance between adjacent fractures $(a / h)$. Due to the periodicity of the configuration, only one crack and the surrounding medium need to be included in the mesh with appropriate periodic boundary conditions applied. The width of the mesh is more than 50 times the crack length to minimize the effects of the far-field lateral boundaries. As shown in Figure 11, the results of the GDC methods (Method B only) are fairly accurate for mode-I with relative errors below $10 \%$. The results for mode-II are less accurate and the most significant factor affecting the accuracy is $h / l_{E}$. When $h / l_{E}=4$ (i.e. eight elements between adjacent cracks), the relative error can be as high as $30 \%$ for large $a / h$ values, but the ascending trend of the $F_{I I^{-}}$ $a /(a+h)$ curve can still be reproduced. When $h / l_{E}=2$, the relative error becomes unacceptably large and fails to represent the general trend of the $F_{I I} a /(a+h)$ curve. Among all the numerical cases, the shortest distance between neighboring cracks is $4 l_{E}$ (i.e. $h / l_{E}=2$ ). If the neighboring cracks are only $2 l_{E}$ apart, Method B for mode-I will fail because all the displacement components used in equation (18) would be zero due to the symmetry of the problem, yielding zero stress intensity. This condition dictates the largest element size that can be used for mode-I.

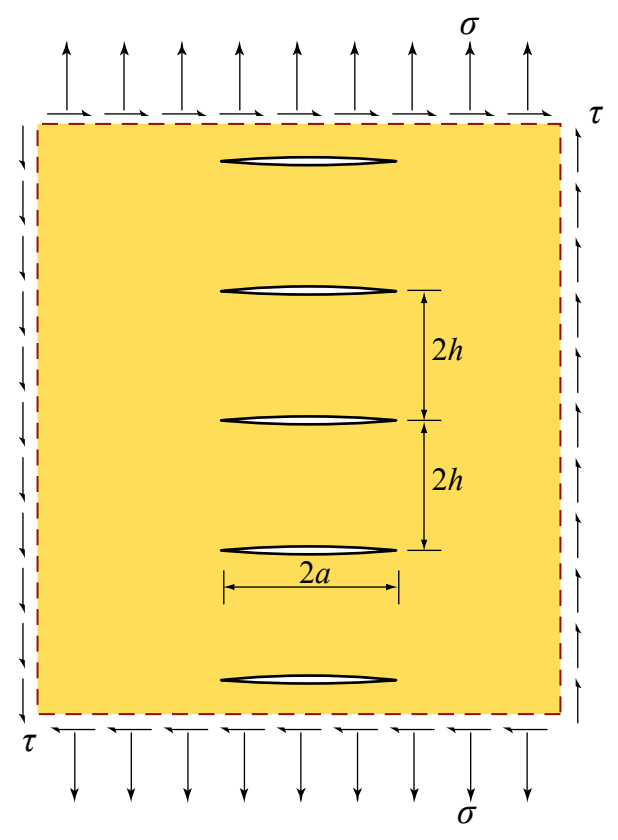

Figure 10 Parallel finite-length fractures in an infinite plane. 

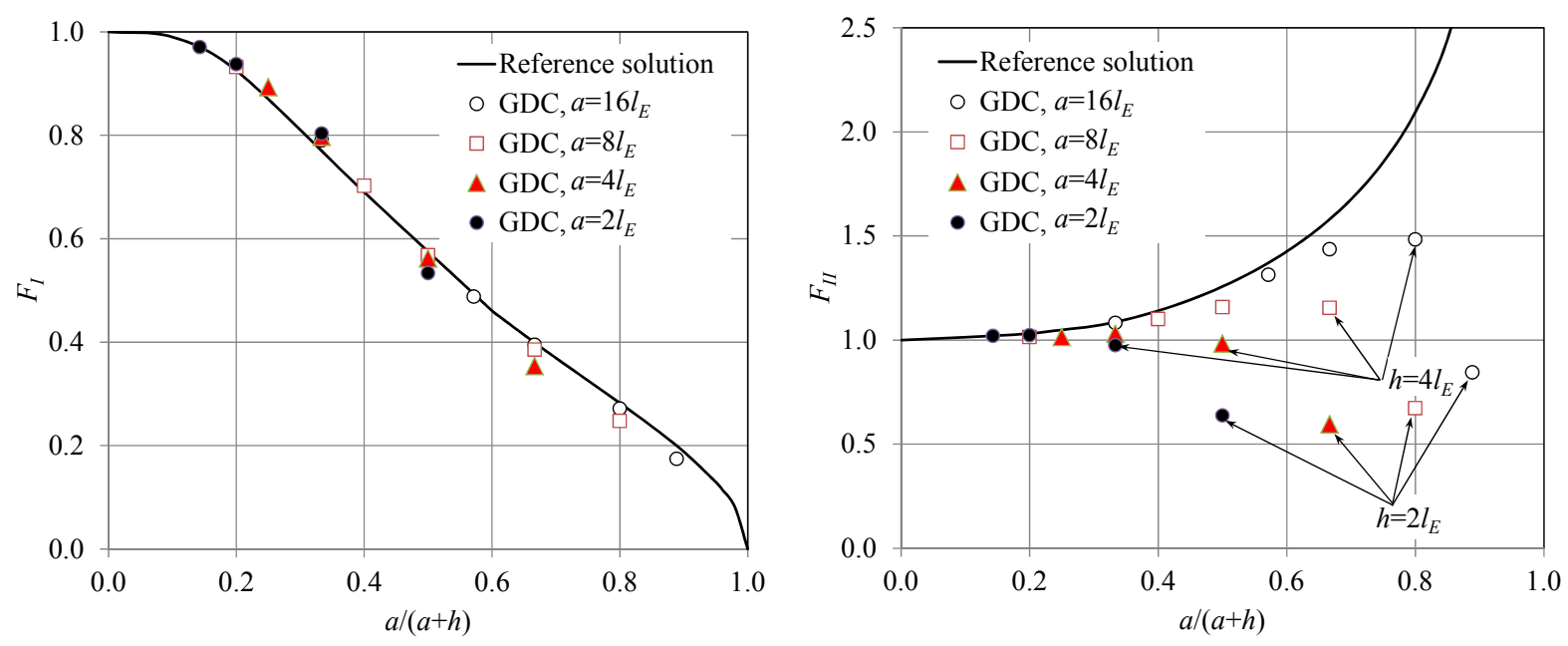

Figure 11 Comparison of the GDC method results and well-accepted reference numerical solutions [1]. The latter are shown as continuous curves and they have an estimated error of less than $1 \%, a /(a+h)$ is used as the horizontal axis to be consistent with the notation in [1]. Note that $a /(a+h)=1 /(1+h / a)$.

6. The effects of mesh configurations and the Poisson's ratio

In all the numerical examples in sections 4 and 5, the Poisson's ratio is assumed to be 0.2 . As shown in equation (1), the Poisson's ratio is related to the value of $\beta$ thereby affecting the neartip displacement field. As mentioned in section 3, the accuracy of the GDC method (without enhancement through the correction multipliers) depends on the ability of the finite element in representing the near-field displacement field. Therefore, it is expected that the values of $C_{I}$ and $C_{I I}$ are dependent on the Poisson's ratio. We repeat the numerical examples on a single fracture in an infinite plane in section 4 with Poisson's ratios ranging from 0 to 0.4 , and the correction multipliers required for obtaining accurate SIF's for different mesh refinement levels are shown in Figure 12. A unified regression model is established by assuming the two constants in equation (20) to vary linearly with respect to the Poisson's ratio, and the regression results are

$$
\begin{aligned}
& C_{I}^{B}=\frac{1.226+0.206 v}{\sqrt{1-(0.349-1.125 v) l_{E} / a}} \\
& C_{I I}^{B}=\frac{1.737-0.048 v}{\sqrt{1-(0.874-0.179 v) l_{E} / a}}
\end{aligned}
$$

The effects of the Poisson's ratio are more significant for mode-I than for mode-II. Even for mode-I, ignoring these effects by using the correction multipliers for $v=0.2$ introduces less than $4 \%$ incremental error to the calculated SIF's for arbitrary Poisson's ratio. 


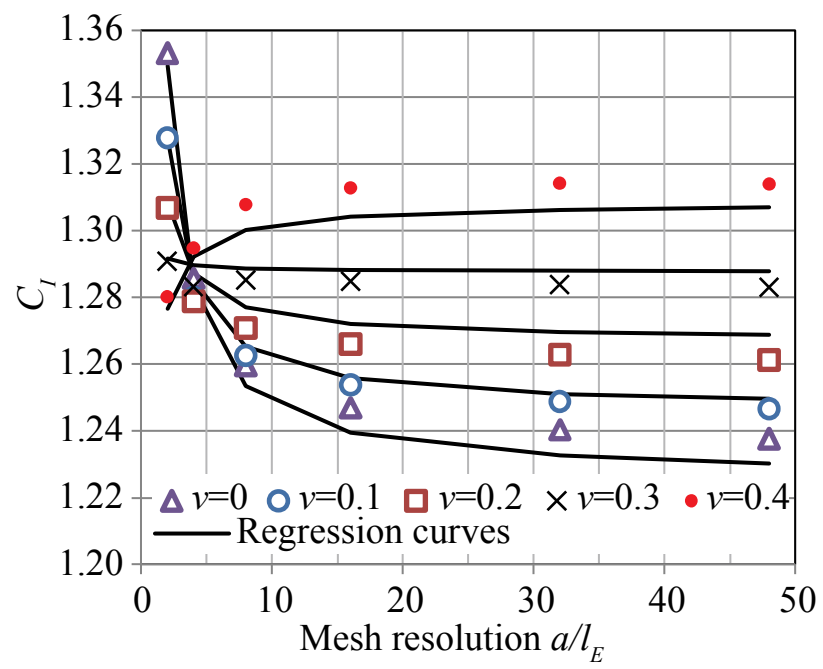

(a)

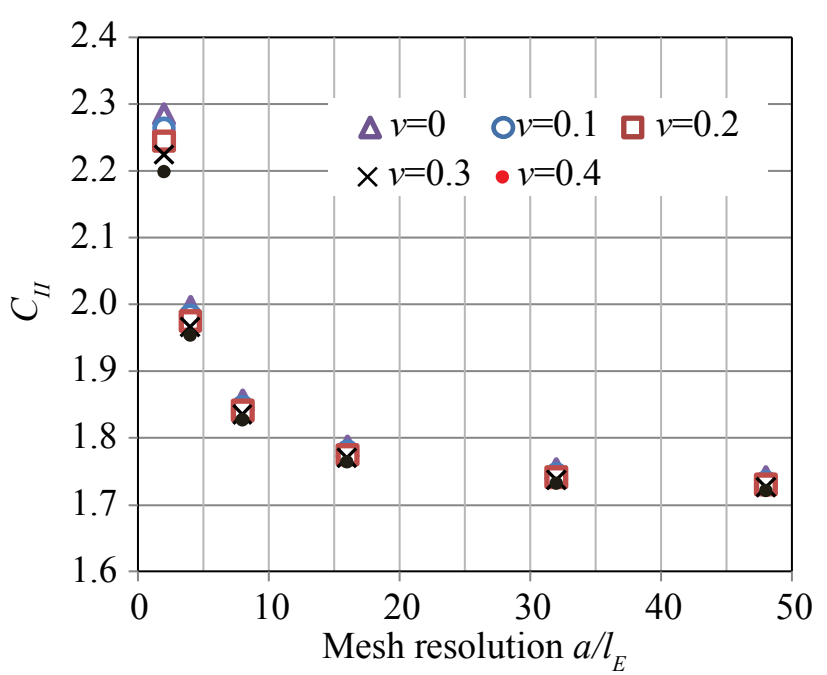

(b)

Figure 12 The effects of the Poisson's on the correction multipliers for (a) mode-I and (b) modeII at different mesh refinement levels. The effects on $C_{I I}$ are small and the regression curves are not plotted. Only the results for Method B are shown.

The correction multipliers are also dependent on the near-tip mesh configuration. All the previous numerical examples are based on the mesh configuration shown in Figure 5(a) where eight triangular elements are connected to the tip node. The other thee configurations in Figure 5 are also common in FEM analysis. We repeat the numerical analysis in section 4 with the additional mesh configurations to determine the correction multipliers for different configurations and the results for a Poisson's ratio of 0.2 are shown in Figure 13. Note that mesh-i, mesh-ii, and mesh-iii use the same space discretization scheme with the only difference among them being in the location of the crack tip and the crack orientation. For a given mesh, the $l_{E}$ value of mesh-iii is $\sqrt{2}$ times larger than that for mesh-i and mesh-ii. To use mesh configuration iv, $l_{E}$ in equation (18) is replaced with $l_{E}{ }^{\prime} \sqrt{3} l_{E} / 2$. This constrains the solution to only use the displacements of points within two element layers of the tip.

The trend of the variation of the correction multipliers with respect to the mesh refinement level is the same for all the mesh configurations. The curves become relatively flat when $a / l_{E}>8$. In configurations $\mathrm{i}$ and iii, the near-tip region is discretized into eight elements in the angular direction while it is discretized into four elements for mesh-ii. Better refinement in the angular direction improves the displacement field representation, yielding correction multipliers closer to unity. In the region with a radius of $2 l_{E}$ around the tip, more elements are involved in mesh-iii than in mesh-i (the mesh is the same for these two configurations but $l_{E}$ for mesh-iii is longer), enabling a better displacement field representation. Despite these observations, the effects of the 
mesh configurations on the correction multipliers are moderate. If we used the correction multipliers for mesh-i on mesh configuration ii, it would induce an error of $4 \%$.

Additionally, though all the examples in this paper are for plane-stress conditions using Method B, application of the generalized Method B to plane-strain conditions or Method A to planestrain and plane-stress conditions is straightforward.
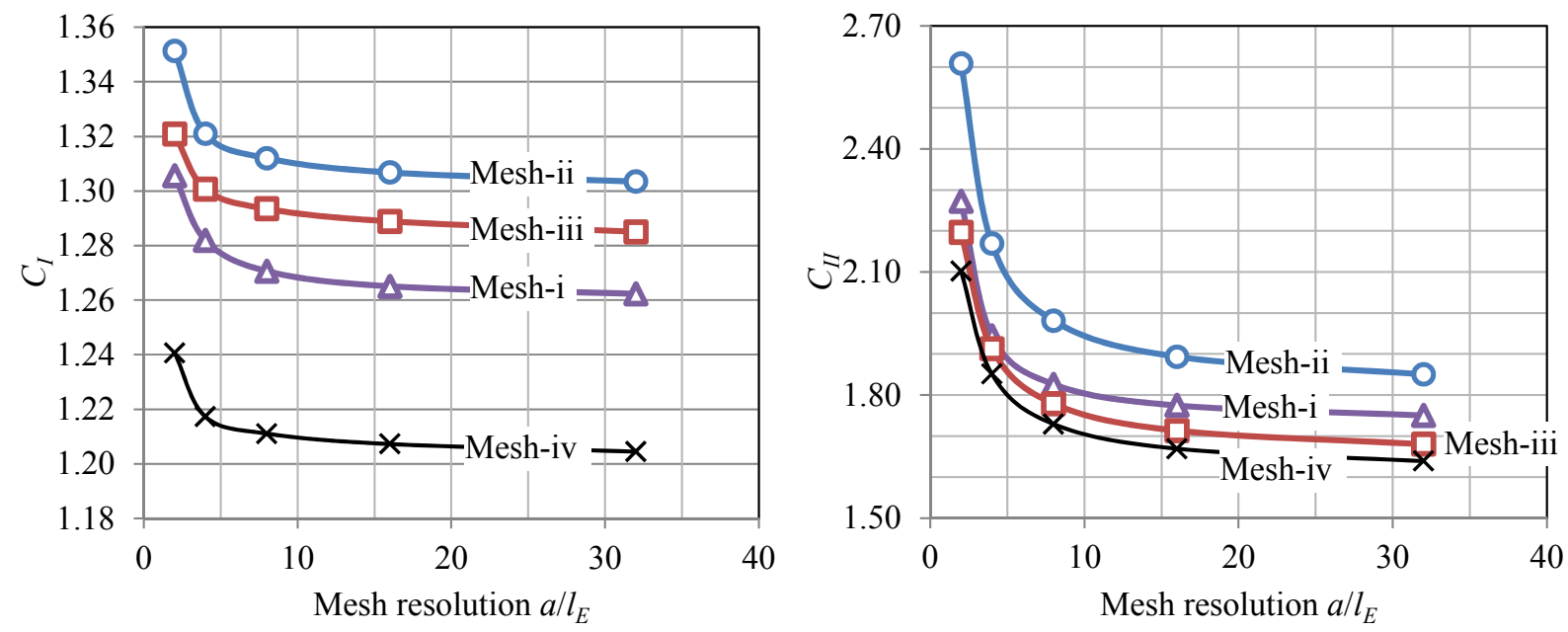

Figure 13 The effects of near-tip mesh configurations on the correction multipliers for (a) mode-I and (b) mode-II at different mesh refinement levels.

\section{Concluding remarks}

The original displacement-based methods for calculating stress intensity factors require quarterpoint finite element elements and near-tip refinement. The generalized displacement correlation (GDC) method proposed in this paper has two advantages: 1) It is designed to work with conventional finite element types, and 2) it uses a homogeneous mesh without local refinement around fracture tips. The former feature makes it convenient to implement the new method in existing finite element packages. The latter is important for modeling dynamic fracture propagation problems where the locations of fractures are not known a priori. The formulation of the new method is also valid for fracture systems where traction and shear exist on the surface of the fractures.

We propose two suites of formulations, termed Method A and Method B, for the GDC method. The former utilizes displacement information within one layer of elements around the fracture tip, and requires quadratic or higher-order finite elements. The latter can work with any element types, but requires displacements within two layers of elements. To enhance accuracy of both methods, a correction multiplier is also proposed. Without this correction term, the accuracy of the GDC method is limited due to the inability of regular finite element types to accurately 
represent the near-tip displacement field. Through a series of numerical examples with a variety of crack configurations, we find that that the new GDC method is acceptably accurate for calculating mode-I stress intensity factors. Even in the limit of mesh coarseness when there is only one element between the two tips of the adjacent fractures, the error is of the order of $10 \%$. The accuracy of Method B for mode-II is less than for mode-I, but acceptable results for most engineering applications, especially for geo-engineering applications, can be obtained even with coarse meshes. Severe errors are inevitable if the points where displacements are used for the calculation are very close to other fracture tips or boundaries of the computation domain. However, this is not unique to the GDC method, and other comparable methods suffer under the same conditions because the near-tip region is inadequately resolved. To correctly model these problems (e.g. tips close to each other or to the boundaries), sufficiently fine meshes must be adopted.

Only the correction multipliers for quadratic six-node triangle elements are presented in this paper. Correction multipliers for any combination of element type and mesh configuration can be easily determined through a small number of FEM simulations following the procedure in section 4. Only one crack-loading configuration needs be considered, and the resultant correction multipliers can be used in arbitrary fracture-load configurations with the same mesh.

\section{Acknowledgments}

This work was performed under the auspices of the U.S. Department of Energy by Lawrence Livermore National Laboratory under Contract DE-AC52-07NA27344. The work of Fu and Carrigan in this paper was supported by the Geothermal Technologies Program of the US Department of Energy, and the work of Johnson and Settgast was supported by the LLNL LDRD project "Creating Optimal Fracture Networks" (\#11-SI-006). This manuscript was approved to be released by LLNL with a release number LLNL-JRNL-501931.

\section{References}

1. Tada H, Paris PC, Irwin GR. The Stress Analysis of Cracks Handbook. NewYork: ASME Press, 2000.

2. Rice JR. A path independent integral and the approximate analysis of strain concentration by notches and cracks. Journal of Applied Mechanics 1968; 35: 379-386, DOI: 10.1.1.160.9792. 
3. Parks DM. A stiffness derivative finite element technique for determination of crack tip stress intensity factors. International Journal of Fracture 1974; 10(4): 487-502, DOI: 10.1007/BF00155252.

4. Chan SK, Tuba IS, Wilson WK. On the finite element method in linear fracture mechanics. Engineering Fracture Mechanics 1970; 2(1):1-17.

5. Banks-Sills L, Sherman D. Comparison of methods for calculating stress intensity factors with quarter-point elements. International Journal of Fracture 1986; 32(2): 127-140.

6. Banks-Sills L, Einav O. On singular, nine-noded, distorted, isoparametric elements in linear elastic fracture mechanics. Computers and Structures 1987; 25(3): 445-449.

7. Zhu WX, Smith DJ. On the use of displacement extrapolation to obtain crack tip singular stresses and stress intensity factors. Engineering Fracture Mechanics 1995; 51(3): 391-400.

8. Barsoum RS. On the use of isoparametric finite elements in linear fracture mechanics. International Journal for Numerical Methods in Engineering 1976; 10(1): 25-37.

9. Shih CF, deLorenzi H, German MD. Crack extension modeling with singular quadratic isoparametric elements. International Journal of Fracture 1976; 12(3): 647-651.

10. Tracey DM. Discussion of 'on the use of isoparametric finite elements in linear fracture mechanics' by R. S. Barsoum. International Journal for Numerical Methods in Engineering 1977; 11(2): 401-402.

11. Li FZ, Shih CF, Needleman A. A comparison of methods for calculating energy release rates. Engineering Fracture Mechanics 1985; 21(2): 405-421.

12. Lim I, Johnston IW, Choi SK. On stress intensity factor computation from the quarter-point element displacements. Communications in Applied Numerical Methods 1992a; 8(5): 291300.

13. Lim I, Johnston IW, Choi SK. Comparison between various displacement-based stress intensity factor computation techniques. International Journal of Fracture 1992b; 58(3):193210.

14. Courtin S, Gardin C, Bezine G, Ben-Hadj-Hamouda H. Advantages of the -integral approach for calculating stress intensity factors when using the commercial finite element software ABAQUS. Engineering Fracture Mechanics 2005; 72(14): 2174-2185.

15. Henshell RD, Shaw KG. Crack tip finite elements are unnecessary. International Journal for Numerical Methods in Engineering 1975; 9(3): 495-507. 
16. Barsoum RS. Triangular quarter-point elements as elastic and perfectly-plastic crack tip elements. International Journal for Numerical Methods in Engineering 1977; 11(1): 85-98.

17. Ingraffea $\mathrm{AR}$, Manu C. Stress-intensity factor computation in three dimensions with quarterpoint elements. International Journal for Numerical Methods in Engineering 1980; 15(10): 1427-1445.

18. Banks-Sills L, Bortman Y. Reappraisal of the quarter-point quadrilateral element in linear elastic fracture mechanics. International Journal of Fracture 1984; 25(3): 169-180.

19. Yehia NAB, Shephard MS. On the effect of quarter-point element size on fracture criteria. International Journal for Numerical Methods in Engineering 1985; 21(10): 1911-1924.

20. Banks-Sills, L. Update: application of the finite element method to linear elastic fracture mechanics. Applied Mechanics Reviews 2010; 63(2), 020803, doi:10.1115/1.4000798.

21. Fu PC, Johnson SM, Carrigan CR. Simulating complex fracture systems in geothermal reservoirs using an explicitly coupled hydro-geomechanical model. Proceedings of the $45^{\text {th }}$ US Rock Mechanics/ Geomechanics Symposium, 11-244, San Francisco, CA, June 26-29, 2011.

22. Guinea GV, Planas J, Elices M. $K_{I}$ evaluation by the displacement extrapolation technique. Engineering Fracture Mechanics 2000; 66(3): 243-255.

23. Williams MD, Jones R, Goldsmith GN. An introduction to fracture mechanics - theory and case studies, Transactions of Mechanical Engineering Vol. ME 14, IEAust, Australia, No. 4, 1989.

24. Harrop LP. The optimum size of quarter-point crack tip element. International Journal for Numerical Methods in Engineering 1982; 18(7): 1101-1103.

25. Srawley JE. Wide range stress intensity factor expressions for ASTM E 399 standard fracture toughness specimens. International Journal of Fracture 1976; 12(3): 475-476. 